\title{
VALIDAÇÃO DA PRACTICE ENVIRONMENT SCALE ENTRE TÉCNICOS E AUXILIARES DE ENFERMAGEM
}

\section{Maria Carolina Pinto Martins, Renata Cristina Gasparino.}

\section{Resumo}

Objective: to validate the Brazilian version of the Practice Environment Scale (PES) between technicians and nursing assistants. Method: a methodological study, carried out in a university hospital in the interior of São Paulo. The sample consisted of 91 professionals who provided direct assistance to the patient and who worked in the unit for more than three months. For data collection, a personal and professional characterization form was used, the Brazilian version of PES, the emotional exhaustion subscale of the Maslach Burnout Inventory, and the job satisfaction and safety climate subscales of the Safety Attitudes Questionnaire Short Form. For reliability assessment, the Cronbach alpha coefficient was calculated and for validity evaluation, the PES subscale scores were correlated with the scores of the other variables, using the Spearman correlation coefficient. Results: all correlations showed significant $(p<0.05)$ and the alpha coefficient reached values equal to or greater than 0.6 in all subscales of the instrument. Conclusion: the Brazilian version of PES between technicians and nursing assistants is a valid and reliable tool to evaluate the characteristics of the nursing work environment.

\section{Palavras-chave:}

Validation studies, Health facility environment, Safety patient.

\section{Introdução}

A equipe de enfermagem constitui a maior parcela de trabalhadores de um hospital, exercendo importante papel em todas as etapas de uma internação hospitalar. Sabe-se que a presença de determinadas características no ambiente de trabalho pode favorecer os resultados com pacientes, profissionais e instituições. A Practice Environment Scale (PES) ${ }^{1}$ pode contribuir para identificar a presença dessas caracterísitcas, entretanto, sua versão brasileira foi validada somente entre enfermeiros e considerando que os técnicos e auxiliares de enfermagem constituem a maior parcela de profissionais da equipe de enfermagem, o objetivo desse estudo foi validar a versão brasileira da PES nesta população.

\section{Método}

Estudo metodológico, realizado em um hospital público e de ensino do interior do estado de São Paulo. A amostra, randomizada, foi composta por 91 técnicos e auxiliares de enfermagem que prestavam atendimento direto ao paciente e trabalhavam na unidade a mais de três meses. Os resultados com os pacientes foram avaliados por meio da percepção da qualidade da assistência prestada e clima de segurança; com os profissionais por meio da percepção sobre a exaustão emocional e satisfação com o trabalho e com relação à instituição, o resultado foi avaliado por meio da intenção do profissional deixar seu emprego no próximo ano.

\section{Resultados}

Tabela 1. Média, desvio-padrão e coeficiente $\alpha$ de Cronbach da versão brasileira da Pratice Environment Scale entre auxiliares e técnicos de enfermagem. Campinas, 2018.

\begin{tabular}{cccc}
\hline PES & Média & DP & a Cronbach \\
\hline Participação & 2,7 & 0,87 & 0,87 \\
Fundamentos & 2,9 & 0,68 & 0,88 \\
Habilidade & 2,7 & 0,81 & 0,86 \\
Adequação & 2,4 & 0,78 & 0,78 \\
Relações & 2,9 & 0,68 & 0,70 \\
\hline
\end{tabular}

Tabela 2. Coeficiente de correlação de Spearman entre a versão brasileira da Pratice Environment Scale entre auxiliares e técnicos de enfermagem, Satisfação com o trabalho e Clima de Segurança, Exaustão Emocional, Percepção da qualidade do cuidado e Intenção de deixar o emprego. Campinas, 2018.

\begin{tabular}{|c|c|c|c|c|c|}
\hline PES & 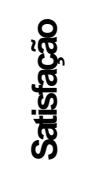 & 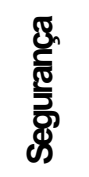 & $\begin{array}{l}\text { 贾 } \\
\frac{7}{3} \\
\text { 离 }\end{array}$ & $\begin{array}{l}\frac{1}{8} \\
\frac{7}{8} \\
\frac{0}{10} \\
0 \\
0\end{array}$ & 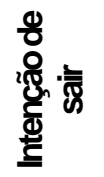 \\
\hline Participação & $0,48^{*}$ & $0,67^{*}$ & $-0,40^{*}$ & $0,25^{* k}$ & $-0,31^{* *}$ \\
\hline Fundamentos & $0,52^{*}$ & $0,57^{\star}$ & $-0,43^{*}$ & $0,34^{* *}$ & $-0,31^{* *}$ \\
\hline Habilidade & $0,53^{*}$ & $0,69^{*}$ & $-0,41^{*}$ & $0,28^{* *}$ & $-0,37^{\star *}$ \\
\hline Adequação & $0,53^{\star}$ & $0,45^{*}$ & $-0,48^{\star}$ & $0,39^{* *}$ & $-0,24^{* *}$ \\
\hline Relações & $0,43^{*}$ & $0,58^{\star}$ & $-0,44^{*}$ & $0,22^{* k}$ & $-0,22^{* *}$ \\
\hline
\end{tabular}

\section{Conclusão}

A versão brasileira da PES entre técnicos e auxiliares de enfermagem é uma ferramenta válida e confiável para avaliar a presença de características que favorecem a prática profissional da enfermagem.

1 Lake ET. The nursing practice environment. Med Care. 2007;64(2):104S-22S. 